\title{
Analysis of the Clinical and Histopathological Patterns of 100 Consecutive Cases of Primary Cutaneous Melanoma and Correlation with Staging
}

\author{
Kyung Wook Nam ${ }^{1}$, Yong Chan Bae ${ }^{1,2}$, Seong Hwan Bae ${ }^{1}$, Kyung Ho Song ${ }^{1}$, Hoon Soo Kim³, \\ Young Jin $\mathrm{Choi}^{4}$ \\ ${ }^{I}$ Department of Plastic and Reconstructive Surgery, Pusan National University School of Medicine, Buasn; ${ }^{2}$ Biomedical Research Institute, \\ Pusan National University Hospital, Buasn; Departments of ${ }^{3}$ Dermatology and ${ }^{4}$ Internal Medicine, Pusan National University School of \\ Medicine, Busan, Korea
}

Background This study analyzed 100 consecutive patients with primary cutaneous melanoma over the course of 13 years to determine whether epidemiological differences correspond to different stages of the disease. We also investigated whether epidemiological characteristics affected the survival rate. Our results were compared with those of selected descriptive studies of melanoma in other East Asian populations, in order to determine whether cutaneous melanoma patterns are similar in East Asian populations.

Methods The patients' medical records were reviewed retrospectively, and we analyzed the relationship of epidemiological characteristics to staging and survival rate. Additionally, papers from Hong Kong and Japan describing these phenomena in East Asian populations were subjected to a statistical comparison.

Results The ratio of males to females was 1:1.8, and the foot was the most frequent tumor site (49\%). Acral lentiginous melanoma occurred most frequently (55\%). Nodular melanoma was associated with a higher stage. Stage III-IV tumors with Clark levels of IV-V were significantly associated with a low survival rate. A statistical analysis of comparable papers reported in Hong Kong and Japan showed similar results with regard to age, tumor location, and histopathological subtypes.

Conclusions This study provides the first full epidemiological description of 100 consecutive cases of primary cutaneous melanoma in Korea, with results similar to those observed in other East Asian populations. Corresponding to previous findings, nodular melanoma tended to occur at a higher stage than other types, and tumors with high Clark levels and high stages showed a lower survival rate.

Keywords Melanoma / Skin neoplasms / Neoplasm staging
Correspondence: Yong Chan Bae Department of Plastic and Reconstructive Surgery, Pusan National University School of Medicine, Biomedical Research Institute, Pusan National University Hospital, 179 Gudeok-ro, Seo-gu, Busan 49241, Korea Tel: +82-51-240-7273

Fax: +82-51-243-9405

E-mail: baeyc2@hanmail.net

Received: 24 Feb 2015• Revised: 11 May $2015 \bullet$ Accepted: 22 May 2015

pISSN: 2234-6163 • elSSN: 2234-6171 • http://dx.doi.org/10.5999/aps.2015.42.6.746• Arch Plast Surg 2015;42:746-752

This work was supported by a clinical research grant from Pusan National University Hospital, 2014.

This article was presented at the Fourth Research and Reconstructive Forum on April 4, 2014 in Busan, Korea.

No potential conflict of interest relevant to this article was reported.

\section{INTRODUCTION}

Malignant melanoma is a form of skin cancer that originates from melanocytes. Several factors, including biological and environmental variables, have been associated with significant differences in incidence rates and clinical features of the disease [1-4]. 
According to previous studies, the incidence rates of malignant melanoma are higher in Western countries than in Eastern countries, and systematic reviews therefore reflect the dynamics of melanoma in Western countries $[5,6]$.

However, it is problematic to apply Western research results to Korea, because malignant melanoma affects different sites and shows different clinical features in Korea. Some systematic reviews on malignant melanoma in South Korea have recently been published; however, an insufficient amount of large-scale studies have been conducted $[7,8]$.

In this study, we selected 100 consecutive patients with primary malignant melanoma in order to reduce selection bias, and performed a retrospective study of clinical and histopathological factors, including sex, age, site, tumor thickness, level of invasion, stage, and histopathological subtype.

Tumor thickness and regional lymph node metastasis are well known to be important prognostic factors of malignant melanoma; however, we attempted to investigate whether other properties are associated with high-stage tumors by examining correlations between the stage of a tumor and other factors. We evaluated differences in staging according to sex, age, location, and histopathological subtype. We also investigated whether epidemiological characteristics were associated with statistically significant difference in the survival rate.

Finally, in order to determine whether relevant clinical features differ among East Asian populations, the results of similar studies dealing with other East Asian populations were compared to our findings using statistical analysis.

\section{METHODS}

We analyzed 100 consecutive primary malignant melanoma patients who underwent surgical treatment at our hospital between September 2000 and November 2013.

\section{Epidemiological characteristics}

The sex and age of each patient, tumor site, tumor thickness, level of invasion, stage, and histopathological subtype of each tumor were analyzed based on the patients' medical records.

Age was classified as under 40 years of age, $40-49$ years, $50-59$ years, $60-69$ years, $70-79$ years, and 80 years and over. The site of the primary tumor was divided into the following four broad areas: the head and neck, the trunk, the upper extremities, and the lower extremities. The upper extremities were subdivided into fingers and other areas. The lower extremities were divided into feet and other locations.

Tumors were divided into five groups according to their Breslow thickness: in situ, $\leq 1 \mathrm{~mm}, 1.01-2.00 \mathrm{~mm}, 2.01-4.00 \mathrm{~mm}$, and $\geq 4.01 \mathrm{~mm}$. The Clark level assesses the depth of melanoma cells in the skin, resulting in the following classifications in situ melanoma limited to the dermis-epidermis junction (Clark level I), melanoma cells invading the papillary dermis but without expansion of this layer (Clark level II), melanoma cells invading and expanding the papillary dermis but not into the reticular dermis (to the interface of the papillary-reticular dermis) (Clark level III), melanoma that has spread into the reticular dermis, but not into the subcutaneous fat (Clark level IV), and melanoma that has invaded the subcutaneous fat or associated subreticular tissues (Clark level V).

In addition, based on the pathological histology of the lesion and the clinical record at the time of diagnosis, melanoma staging was performed according to the 2013 Guidelines of the $\mathrm{Na}$ tional Comprehensive Cancer Network.

The histopathological subtypes of melanoma are lentigo maligna melanoma, superficial spreading melanoma, acral lentiginous melanoma, and nodular melanoma.

\section{Correlation between epidemiological characteristics and staging}

The Mann-Whitney U test was used to compare the relationship between sex and staging, whereas the Kruskal-Wallis test was used to determine the relationships of age, site, and histopathological subtype with staging. P-values $<0.05$ were considered to indicate statistical significance. For factors shown to have a statistically significant relationship to staging, the MannWhitney $U$ test was performed for comparison between characteristics, followed by the Bonferroni correction. P-values $<0.008$ were considered to indicate statistical significance.

\section{Survival rate}

We assessed the survival of the patients by checking their recent medical records and through telephone contact. The mean follow-up period was 66 months (range, 24-168 months). The influence of prognostic factors on survival were assessed by the Cox proportional hazard model. First, univariate analyses were performed with gender, age, staging, tumor location, depth of invasion, thickness of lesion, and clinical pathological classification. We estimated the survival curve using the Kaplan-Meier method (log-rank test), which calculates the survival rate from the start of the study to patients' deaths or censoring, and created a graphic visualization of this curve. Statistical analyses were performed using the SPSS ver. 12.0 (SPSS Inc., Chicago, IL, USA).

\section{Epidemiological characteristics among East Asian populations}

Subsequently, we performed a literature search on PUBMED 
for clinical research on malignant melanoma in East Asian populations, and examined 10 selected studies in order to determine whether melanoma in East Asian populations presents with similar clinical features.

After a review of the selected articles, we chose those published by Luk et al. [9] in Hong Kong and Ishihara et al. [10] in Japan for more detailed analysis, because they appeared to be the most reliable and used the same classification criteria.

Data collected from those articles were statistically analyzed and compared with our research data to determine whether East Asians have similar clinical features of malignant melanoma.

The chi-squared test was used, and P-values $<0.05$ were considered to indicate statistical significance.

\section{RESULTS}

\section{Epidemiological characteristics}

Our sample included 64 females and 36 males, with a 1:1.8 male-to-female ratio.

The average age of the patients at the time of diagnosis was 55 years old (range, 21-83 years). The majority of the patients (34) were in their 60s, 22 were in their 70 s, and 17 were in their 50 s (Table 1).

The most common site of primary tumors was the lower ex-

Table 1. Distribution of patients by age according to the stage of the tumor

\begin{tabular}{|lcccccc|}
\hline Age (yr) & Stage 0 & Stage I & Stage II & Stage III Stage IV & Total \\
\hline$\leq 39$ & 0 & 2 & 2 & 5 & 0 & 9 \\
$40-49$ & 2 & 5 & 2 & 4 & 0 & 13 \\
$50-59$ & 5 & 4 & 1 & 7 & 0 & 17 \\
$60-69$ & 4 & 6 & 7 & 15 & 2 & 34 \\
$70-79$ & 1 & 3 & 12 & 5 & 1 & 22 \\
$\geq 80$ & 0 & 0 & 1 & 2 & 2 & 5 \\
Total & 12 & 20 & 25 & 38 & 5 & 100 \\
\hline
\end{tabular}

Table 2. Anatomical distribution of malignant melanoma according to the stage of the tumor

\begin{tabular}{|lcccccr|}
\hline Site & Stage & Stage I & Stage II & Stage III & Stage IV Total \\
\hline Head and neck & 1 & 4 & 2 & 4 & 0 & 11 \\
Upper extremity & & & & & & \\
$\quad$ Finger & 3 & 2 & 2 & 4 & 0 & 11 \\
Other & 0 & 2 & 2 & 2 & 0 & 6 \\
$\quad$ Subtotal & 3 & 4 & 4 & 6 & 0 & 17 \\
Lower extremity & & & & & & \\
$\quad$ Foot & 7 & 9 & 13 & 15 & 5 & 49 \\
Other & 1 & 0 & 3 & 6 & 0 & 10 \\
$\quad$ Subtotal & 8 & 9 & 16 & 21 & 5 & 59 \\
Trunk & 0 & 3 & 3 & 7 & 0 & 13 \\
Total & 12 & 20 & 25 & 38 & 5 & 100 \\
\hline
\end{tabular}

tremities (60 cases), including the feet ( 49 cases), followed by 18 cases in the upper extremities, 11 cases in the trunk, and 11 cases in the head and neck (Table 2).

Measuring the Breslow thickness showed that 12 of the 100 cases involved in situ melanoma. The Breslow thickness was $\leq 1$ $\mathrm{mm}$ in 15 patients, $1.01-2.00 \mathrm{~mm}$ in 13 patients, $2.01-4.00 \mathrm{~mm}$ in 28 patients, and $\geq 4.01 \mathrm{~mm}$ in 32 patients (Table 3 ).

Based on the level of invasion, the largest number of cases (38), had Clark level IV, 14 cases were Clark level I, 10 cases were Clark level II, 15 cases were Clark level III, and 23 cases were Clark level V (Table 4).

Staging was found to be fairly evenly distributed, with 12 cases in stage 0,17 cases in stage I, 25 cases in stage II, 38 cases in stage III, and 5 cases in stage IV. Of the primary tumors, a plurality (32) were T4. T4 is used to indicate melanoma with a thickness $>4.0 \mathrm{~mm}$ regardless of whether an ulcer is present.

With regard to regional lymph node involvement, 38 tumors were classified as N1, and a range of regional lymph nodes were found to be involved. Distant metastasis (M1) was found in four cases.

Regarding histopathological subtypes, the largest group (58 cases) was composed of acral lentiginous melanoma, followed by nodular melanoma (29 cases), superficial spreading melanoma ( 9 cases), and lentigo maligna melanoma ( 4 cases) (Table 5 ).

\section{Correlations between epidemiological characteristics and staging}

No significant relationships were observed between sex and staging $(\mathrm{P}=0.437)$, age and staging $(\mathrm{P}=0.391)$, and location and staging $(\mathrm{P}=0.420)$. However, a significant relationship was

Table 3. Distribution of patients by thickness of melanoma

\begin{tabular}{|lc|}
\hline Tumor thickness & No. of patients \\
\hline In situ & 12 \\
$\leq 1 \mathrm{~mm}$ & 15 \\
$1.01-2.00 \mathrm{~mm}$ & 13 \\
$2.01-4.00 \mathrm{~mm}$ & 28 \\
$\geq 4.01 \mathrm{~mm}$ & 32 \\
Total & 100 \\
\hline
\end{tabular}

Table 4. Distribution of patients by Clark level

\begin{tabular}{|lc|}
\hline Clark level & No. of patients \\
\hline I & 14 \\
II & 10 \\
III & 17 \\
IV & 36 \\
V & 23 \\
Total & 100 \\
\hline
\end{tabular}


Table 5. Distribution of patients by histopathological subtype according the staging distribution

\begin{tabular}{|lcccccr|}
\hline Value & Stage 0 & Stage I & Stage II & Stage III Stage IV Total \\
\hline $\begin{array}{c}\text { Acral } \\
\quad \text { lentiginous }\end{array}$ & 10 & 11 & 14 & 10 & 13 & 58 \\
$\begin{array}{l}\text { Lentigo } \\
\quad \text { maligna }\end{array}$ & 1 & 1 & 2 & 0 & 0 & 4 \\
$\begin{array}{l}\text { Nodular } \\
\text { Superficial }\end{array}$ & 0 & 4 & 6 & 12 & 7 & 29 \\
$\quad$ spreading & 1 & 3 & 3 & 0 & 2 & 9 \\
Total & 12 & 19 & 25 & 22 & 22 & 100 \\
\hline
\end{tabular}

\section{Table 6. Statistical analysis of histopathological subtypes}

\begin{tabular}{|lc|}
\hline Value & P-value $^{\text {a) }}$ \\
\hline A vs. L & 0.324 \\
A vs. N & $0.003^{\star}$ \\
A vs. S & 0.512 \\
L vs. N & 0.041 \\
L vs. S & 0.456 \\
N vs. S & $0.008^{\star}$ \\
\hline
\end{tabular}

A, acral lentiginous; L, lentigo maligna; $N$, nodular; $S$, superficial spreading. a)Mann-Whitney U test with Bonferroni correction; *Statistically significant with P-value $<0.008$.

observed between histopathological subtype and staging $(\mathrm{P}=$ 0.05). Nodular melanoma was associated with a higher stage than acral lentiginous melanoma $(\mathrm{P}=0.003)$ and superficial spreading melanoma $(\mathrm{P}=0.008)$ (Table 6).

\section{Survival rate}

Table 7 shows the survival rate. Of the patients included in our sample, 20 died of malignant melanoma, five died of other diseases, and 20 were lost to follow-up.

\section{Sex and age}

Based on the hazard ratio of men and women for mortality, men were twice as likely to die (0.492), but no difference in the survival rate was found $(\mathrm{P}=0.123)$. Similarly, age was not associated with a different survival rate. In the Kaplan-Meier analysis, the differences in the survival rate among subgroups defined according to sex and age were not significant.

\section{Clinical staging}

The hazard ratio for mortality associated with stage III-IV tumors was approximately three times higher compared to tumors with a lower stage, which was a statistically significant relationship $(P=0.034)$. In the Kaplan-Meier analysis, significant differences in the survival rate between these two groups were observed.
Table 7. Univariate analysis using the Cox proportional hazard model

\begin{tabular}{|c|c|c|c|}
\hline Characteristic & $\begin{array}{l}\text { Mortality } \\
(\%)\end{array}$ & P-value & $\begin{array}{l}\text { Hazard ratio (95\% } \\
\text { confidence interval) }\end{array}$ \\
\hline Sex & & 0.123 & \\
\hline Male & 10/36 (27.8) & & 1 \\
\hline Female & 9/64 (14.1) & & $0.492(0.200-1.211)$ \\
\hline \multicolumn{4}{|l|}{ Age (yr) } \\
\hline$<55$ & 5/28 (19.2) & 0.888 & 1 \\
\hline $55-64$ & 6/31 (21.7) & 0.886 & $0.917(0.279-3.012)$ \\
\hline$\leq 65$ & 8/41 (18.6) & 0.765 & $1.187(0.387-3.641)$ \\
\hline \multicolumn{4}{|l|}{ Location } \\
\hline Head and neck & 4/11 (36.4) & 0.267 & 1 \\
\hline Upper extremities & 2/18 (11.1) & 0.125 & $0.263(0.048-1.448)$ \\
\hline Lower extremities & $1 / 60(16.7)$ & 0.076 & $0.346(0.107-1.118)$ \\
\hline Trunk & $3 / 11(27.3)$ & 0.485 & $0.584(0.129-2.646)$ \\
\hline \multicolumn{4}{|l|}{ Histology } \\
\hline Acral lentiginous & 9/58 (15.5) & 0.660 & 1 \\
\hline Lentigo maligna & $0 / 4(0.0)$ & 0.993 & $0.000(0.000-0.000)$ \\
\hline Nodular & 8/29 (27.6) & 0.212 & $1.834(0.707-4.757)$ \\
\hline Superficial spreading & 2/9 (22.2) & 0.866 & $1.141(0.246-5.296)$ \\
\hline Clinical stage & & $0.034^{\star}$ & \\
\hline $0-\|$ & $7 / 56(12.5)$ & & 1 \\
\hline III-IV & $12 / 44(27.3)$ & & 2.601 (1.037-6.523) \\
\hline Clark level & & $0.031^{*}$ & \\
\hline |-III & $3 / 41(7.3)$ & & 1 \\
\hline IV-V & $16 / 59(27.1)$ & & $3.883(1.131-13.336)$ \\
\hline Breslow & & 0.075 & \\
\hline$\leq 2 \mathrm{~mm}$ & $4 / 40(10.0)$ & & 1 \\
\hline$>2 \mathrm{~mm}$ & $15 / 60(25.0)$ & & $2.728(0.905-8.222)$ \\
\hline
\end{tabular}

\section{Site of the primary tumor}

The mortality rate associated with head and neck tumors was higher, but this finding was not statistically significant $(\mathrm{P}=$ 0.267). In the Kaplan-Meier analysis, no significant differences were found in the survival rate depending on the primary tumor site.

\section{Level of invasion}

The hazard ratio for mortality associated with Clark level IV-V tumors was approximately four times higher than in tumors with a lesser level of invasion. This difference was statistically significant $(\mathrm{P}=0.031)$. In the Kaplan-Meier analysis, a significant difference in the survival rate depending on level of invasion was found.

\section{Tumor thickness}

No significant difference was found in the survival rate between tumors with a thickness $<2 \mathrm{~mm}$ and those with a thickness $>2$ $\mathrm{mm}(\mathrm{P}=0.075)$. In the Kaplan-Meier analysis, no significant difference in the survival rate depending on tumor thickness was found. 
Table 8. Distribution of malignant melanoma patients by age, tumor site, and tumor subtype in Pusan, Hong Kong, and Japan

\begin{tabular}{|c|c|c|c|c|}
\hline Characteristic & $\begin{array}{c}\text { Pusan } \\
(n=100)\end{array}$ & $\begin{array}{l}\text { Hong Kong } \\
\quad(n=63)\end{array}$ & $\begin{array}{c}\text { Japan }^{\text {b) }} \\
(n=642)\end{array}$ & P-value \\
\hline \multicolumn{4}{|l|}{ Age (yr) } & 0.066 \\
\hline$\leq 49$ & $22(22.0)$ & $14(22.2)$ & $194(30.2)$ & \\
\hline $50-59$ & $17(17.0)$ & $13(20.6)$ & 118 (18.4) & \\
\hline $60-69$ & $34(34.0)$ & $20(31.7)$ & $131(20.4)$ & \\
\hline $70-79$ & $22(22.0)$ & $11(17.5)$ & $142(22.1)$ & \\
\hline$\leq 80$ & $5(5.0)$ & $5(7.9)$ & $57(8.9)$ & \\
\hline \multicolumn{4}{|l|}{ Site } & 0.071 \\
\hline Head and neck & $11(11.0)$ & $7(11.1)$ & $96(15.0)$ & \\
\hline Upper extremity & $18(18.0)$ & $5(7.9)$ & $142(22.1)$ & \\
\hline Lower extremity & $60(66.0)$ & 45 (71.4) & $308(48.0)$ & \\
\hline Trunk & $11(11.0)$ & $6(9.5)$ & $96(15.0)$ & \\
\hline \multicolumn{4}{|l|}{ Subtype } & 0.273 \\
\hline Acral lentiginous & $58(58.0)$ & $32(50.8)$ & $308(48.0)$ & \\
\hline Lentigo maligna & $4(4.0)$ & 2 (3.2) & 46 (7.2) & \\
\hline Nodular & $29(29.0)$ & $17(27.0)$ & $172(26.8)$ & \\
\hline Superficial spreading & $9(9.0)$ & $12(19.0)$ & $116(18.1)$ & \\
\hline \multicolumn{5}{|c|}{ 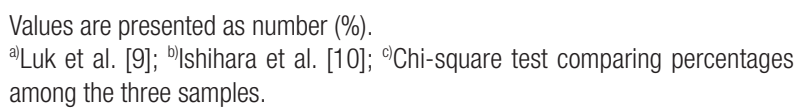 } \\
\hline
\end{tabular}

\section{Histopathological subtype}

No significant differences in survival according to the clinical pathological classification were found $(\mathrm{P}=0.660)$. In the Kaplan-Meier analysis, the differences in the survival rate depending on the clinical pathological classification were not significant.

\section{Epidemiological characteristics of East Asian populations}

The results of the statistical analysis comparing the findings of Luk et al. [9] in a population from Hong Kong and Ishihara et al. $[10]$ in a Japanese population with the results of this study showed no significant differences in age $(\mathrm{P}=0.066)$, site $(\mathrm{P}=$ $0.071)$, and histopathological subtype $(\mathrm{P}=0.273)$ (Table 8$)$.

\section{DISCUSSION}

Despite the increasing number of studies that have recently been conducted on malignant melanoma, an insufficient amount of studies adequately differentiate between primary or recurrent malignant melanoma, whether consecutive patient groups are studied, or include a sufficient quantity of cases of malignant melanoma. Therefore, in this study, we attempted to increase the statistical robustness of our findings by targeting 100 consecutive patients with primary malignant melanoma. In these 100 cases, either wide resection or sentinel lymph node dissection was performed, and all peripheral margins tested negative.
In this study, the sex ratio of new patients with cutaneous melanoma favored female patients (1:1.8 male-to-female ratio), unlike what has been observed in Korean reports that were similarly designed or that deliberately included more men [11-13].

The average age of patients with cutaneous melanoma was 55 years, corresponding to the results of other studies conducted in South Korea, in which the average age has tended to be in the late 50s $[8,11,12]$.

In our sample of 100 cases, we found that the most common site of primary malignant melanoma was the lower extremities (61\%), particularly the feet (49\%). Other studies published in South Korea have reported similar results.

In our study, the histopathological subtypes of cutaneous melanoma included acral lentiginous melanoma (58/100), nodular melanoma (29/100), and superficial spreading melanoma (9/ 100). Won et al. [14], Lee et al. [11], and Chun et al. [13] likewise reported acral lentiginous melanoma to be the most common subtype (in $62.1 \%, 56.9 \%$, and $63.6 \%$ of cases, respectively).

A comparative statistical analysis of Luk et al. [9] and Ishihara et al. [10] was possible since they used the same criteria. Statistical analysis performed to investigate epidemiological characteristics among East Asian populations based on age, site, and histopathological subtype showed no significant differences between each of those two studies and our findings, confirming that similar epidemiological characteristics can be found among East Asian populations. Some papers have investigated large samples of cases in China and Japan. We reviewed 10 potentially relevant papers and attempted to increase the applicability of our statistical findings by comparing them to papers that used clear classifications. However, this was not possible for all papers; for example, the study of Chi et al. [15] included 522 cases that were not yet classified, and it was therefore difficult to obtain accurate statistics. Moreover, we hoped to compare our findings with those of other studies evaluating the Korean population, but could not perform a comparative statistical analysis of this type due to the insufficient quantity of papers and their non-standardized classification of tumors.

A similar male-to-female ratio was found to studies conducted in Western countries $[5,16]$. The prevalence of malignant melanoma increases with increasing age. According to the age distribution, melanoma is more common in patients in their 60s and 70 s than in patients in their 50 s. The most common sites of primary melanoma among white men have been found to be the trunk, head, and neck, and the lower extremities have been found to be the most common site for women, while a low incidence of melanoma on the hands and feet has been observed in this population. In comparison, black Africans, African-Americans, and Asians have a tendency for primary melanoma to oc- 
cur on the more distal parts of the upper and lower limbs [1-4]. According to a clinical histopathological analysis, in Western countries, the subtypes of melanoma occur in the following order: superficial spreading melanoma (70\%), nodular melanoma (15\%), lentigo maligna melanoma (10\%), and acral lentiginous melanoma (5\%). These results differ significantly from those of our study. In the future, substantial research on racial differences in the presentation of melanoma will be required.

According to our analysis of the relationships of sex and age with the site of the primary tumor and the histopathological subtype, no significant relationship was observed between sex and staging. Garbe and Leiter [17] reported that the survival rate of women was higher than that of men, even if the thickness of the tumor had changed.

No significant relationship was observed between age and staging in our study, but Clark et al. [18] reported that the prevalence of malignant melanoma increased with age and found melanoma to have poor prognosis. No significant relationship was observed between the stage and the site of the primary tumor. However, Jang et al. [7] found that a higher five-year survival rate was associated with primary lesions on the upper extremities, back, and feet, whereas another study reported high mortality rates in patients with melanoma occurring on the back, thorax, upper arm, neck, and scalp. In addition, Krementz et al. [19] reported that patients with melanoma on the fingers and toes had a lower five-year survival rate (27\%).

In contrast to these results, Chamberlain et al. [20] reported that age, sex, and site were not related to the overall survival rate, but that staging at the time of diagnosis was higher in men than in women.

With regards to the clinical pathological classification, nodular melanoma showed was associated with a higher stage than superficial spreading melanoma and acral lentiginous melanoma. This result was found to be statistically significant. Many studies have reported that vertical growth of nodular melanoma is associated with a poor prognosis $[7,8,21]$, which corresponds to the results of this study, in which nodular melanoma was associated with a relatively high stage.

However, Shaw et al. [22] reported that the prognosis of nodular melanoma was similar to that of other subtypes, adjusting for other risk factors such as tumor thickness. Jang et al. [7] also reported more findings of stage III and IV in cases of nodular melanoma than other stages, but no difference in survival rate was found and the histopathological subtype was not related to the prognosis. Similarly, the current study did not show a statistically significant difference in the survival rate depending on these factors.

No statistically significant difference was found between the stages of lentigo maligna melanoma and the stages of nodular melanoma. However, future comparison is needed, since the number of patients was not sufficient for a proper comparison. Other studies have shown that nodular melanoma is associated with a poorer prognosis than lentigo maligna melanoma. Moreover, a study has found that lentigo maligna melanoma is the least aggressive type of melanoma. Based on this, we expect nodular melanoma to be associated with a higher stage than lentigo maligna melanoma.

Clark level and stage were associated with the survival rate. In particular, stage III-IV tumors with a Clark level of IV-V were associated with a significantly lower survival rate than that observed for other tumors.

Many Korean studies have shown that clinical staging has a significant impact on survival, so it is probable that research on the characteristics of high-stage tumors will have significant clinical implications.

This study is expected to provide useful data for future studies of malignant melanoma in Koreans and East Asians more broadly.

In this study, we investigated the clinical and histopathological findings of 100 consecutive patients with primary malignant melanoma who underwent surgical treatment. One major result was that the most common anatomical site of the primary tumor was the foot. Acral lentiginous melanoma was the most common type. Moreover, sex, age, and tumor location showed no relationship with staging. Nodular melanoma tended to occur at a higher stage than acral lentiginous melanoma and superficial spreading melanoma. The Clark level and stage were found to affect mortality; in particular, stage III-IV tumors with a Clark level of IV-V were associated with high mortality rates.

Furthermore, malignant melanoma is known to have race-specific patterns of presentation. A comparison of our results to those of studies reporting on populations from Hong Kong and Japan showed that similar dynamics of malignant melanoma occur in East Asian populations.

\section{REFERENCES}

1. Reintgen DS, McCarty KM Jr, Cox E, et al. Malignant melanoma in black American and white American populations: a comparative review. JAMA 1982;248:1856-9.

2. Kukita A, Ishihara K. Clinical features and distribution of malignant melanoma and pigmented nevi on the soles of the feet in Japan. J Invest Dermatol 1989;92:210S-213S.

3. Collins RJ. Melanoma in the Chinese of Hong Kong: emphasis on volar and subungual sites. Cancer 1984;54:1482-8.

4. Choi SJ, Bae YC, Moon JS, et al. An analysis of clinical and 
histopathological pattern of malignant melanoma. J Korean Soc Plast Reconstr Surg 2007;34:557-61.

5. Lipsker D, Engel F, Cribier B, et al. Trends in melanoma epidemiology suggest three different types of melanoma. $\mathrm{Br} \mathrm{J}$ Dermatol 2007; 157:338-43.

6. Kuphal S, Bosserhoff A. Recent progress in understanding the pathology of malignant melanoma. J Pathol 2009;219: 400-9.

7. Jang KA, Kim JH, Choi JH, et al. A clinico-histopathological study of malignant melanoma. Korean J Dermatol 2000; 38:1435-43.

8. Park KD, Lee SJ, Lee WJ, et al. Clinicopathological Features of Cutaneous Malignant Melanoma. Korean J Dermatol 2007;45:149-58.

9. Luk NM, Ho LC, Choi CL, et al. Clinicopathological features and prognostic factors of cutaneous melanoma among Hong Kong Chinese. Clin Exp Dermatol 2004;29:600-4.

10. Ishihara K, Saida T, Yamamoto A, et al. Updated statistical data for malignant melanoma in Japan. Int J Clin Oncol 2001;6:109-16.

11. Lee DH, Kim YC, Cho SH, et al. Clinicopathologic analysis of malignant melanoma. Korean J Dermatol 2002;40:91423.

12. Park DH, Seo SJ, Park MC, et al. Clinical study of malignant melanoma for recent 14 years. J Korean Soc Plast Reconstr Surg 2009;36:299-305.

13. Chun JS, Yun SJ, Lee JB, et al. A statistical observation on 1,430 cases of cutaneous malignant tumors over 20 years (1987-2006) in Gwangju City and Chonnam Province. Ko- rean J Dermatol 2009;47:667-73.

14. Won YH, Hur SG, Lee SC, et al. Clinical and histopathologic study of malignant melanoma (1980-1990.4). Korean J Dermatol 1991;29:193-202.

15. Chi Z, Li S, Sheng X, et al. Clinical presentation, histology, and prognoses of malignant melanoma in ethnic Chinese: a study of 522 consecutive cases. BMC Cancer 2011;11:85.

16. Chifu MD, Sin A, Chifu M, et al. Primary cutaneous melanoma: 7-year follow-up (2003-2009). Acta Med Marisiensis 2011;57:25-7.

17. Garbe C, Leiter U. Melanoma epidemiology and trends. Clin. Dermatol 2009;27:3-9.

18. Clark WH Jr, From L, Bernardino EA, et al. The histogenesis and biologic behavior of primary human malignant melanomas of the skin. Cancer Res 1969;29:705-27.

19. Krementz ET, Feed RJ, Coleman WP 3rd, et al. Acral lentiginous melanoma: a clinicopathologic entity. Ann Surg 1982; 195:632-45.

20. Chamberlain AJ, Fritschi L, Giles GG, et al. Nodular type and older age as the most significant associations of thick melanoma in Victoria, Australia. Arch Dermatol 2002;138: 609-14.

21. Chen YJ, Wu CY, Chen JT, et al. Clinicopathologic analysis of malignant melanoma in Taiwan. J Am Acad Dermatol 1999;41:945-9.

22. Shaw HM, Balch CM, Soong SJ, et al. Prognostic histopathological factors in malignant melanoma. Pathology 1985;17: $271-4$. 